\title{
Chinas Aufstieg und geopolitische Herausforderungen für die transatlantische Gemeinschaft
}

\author{
Katharina Götsch*
}

\begin{abstract}
China's fast-paced economic development and its rise as the global economic power house in the past decades awoke fears - and in part also perceptions of threat - among Western countries, especially the United States which has been afraid of international decline and internal weakening several times before. While trying to force China into playing a more responsible and constructive role within the international community (e.g. on issues such as climate and environmental agreements, nuclear weapons, international sanctions, etc.), the West also aims at containing China's military, diplomatic and economic expansion and influence on a regional and global scale. Building on theoretical discourses and political analyses, this article examines the geopolitical power shift among the United States, Europe and Asia caused by China's rise on the basis of empirical examples. The central research question addresses the consequences and effects of global power shifts within the international system (from the West to the East) for the transatlantic partnership on the one hand and the global position of Europe and the United States on the other. Thus, possible strategies of Western players for dealing with the challenges resulting from China's growing importance and the impacts on international relations are described and analyzed.
\end{abstract}

Keywords: Geopolitics, international relations, China, United States, Europe, transatlantic partnership

Stichworte: Geopolitik, international Beziehungen, China, USA, Europa, transatlantisches Bündnis

\section{Chinas Aufstieg zum Global Player}

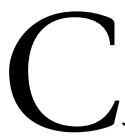

hinas rasanter ökonomischer Aufstieg seit der Öffnung des Landes unter Deng Xiaoping in den späten 1970er Jahren (vgl. Kissinger 2011, Seitz 2000, Lee 2011 u.a.) führt seit mehreren Jahren zu verstärkten, teils überaus emotional geführten Debatten und politischen Auseinandersetzungen in der internationalen Gemeinschaft, insbesondere in den USA und in Europa. Als mittlerweile zweitgrößte Volkswirtschaft weckt China zugleich Erwartungen und Befürchtungen in Bezug auf seine globale Rolle und seine vermeintlichen geografischen, politischen und militärischen Expansionstendenzen (vgl. Shambaugh 2013, Kissinger 2011, u.v.a.). Seit Ende der 1990er Jahre wird der wirtschaftliche und militärische Aufstieg Chinas im Westen zusehends mit Argwohn betrachtet. Insbesondere in den USA wachsen Sorgen, durch das hohe Handelsdefizit in ein Abhängigkeitsverhältnis geraten zu sein oder den Status als Supermacht an den asiatischen Riesen zu verlieren. Auch der wachsende Einfluss Chinas in der pazifischen Region und seine jährlich steigenden Militärausgaben werden von einigen Kräften als geopolitische Bedrohung und Gefährdung für den amerikanischen Führungsanspruch angesehen (vgl. Tisdall 2014, Ross 2005, u.a.).

Auch Europa setzt sich verstärkt mit den durch Chinas wirtschaftliche Expansion entstehenden Konfliktpunkten auseinander. Im Gegensatz zu den USA steht auf dem Kontinent allerdings nicht die Hegemoniefrage bzw. die regionale Machtstellung Chinas im Mittelpunkt des Diskurses. Handelskonflikte, Rüstungskontrolle und multilaterale Umweltverträge sind für die europäisch-chinesischen Beziehungen von größerer Bedeutung (vgl. Fröhlich 2012).

Daraus folgt die Frage, welche Verschiebungen und Herausforderungen sich in dieser Konstellation für die transatlantische Partnerschaft aus geopolitischer Sicht ergeben. Kurz nach dem Platzen einer Immobilien- und Kreditblase im Herbst 2007 in den USA und der darauf folgenden Finanz- und Wirtschaft- krise in den westlichen Industrieländern schien die globale Überlegenheit und Vorherrschaft des westlichen, von den USA geführten Modells schlagartig zu bröckeln. Banken und Versicherungen kollabierten in einem Land nach dem anderen wie Kartenhäuser. Das transatlantische Verhältnis war seit der Irak-Invasion der Bush-Administration und ihrer Umgehung des UN-Sicherheitsrats bereits stark abgekühlt. Die missionarische Sicherheits- und Außenpolitik der USA unter George W. Bush nach dem Terroranschlag des 11. September 2001 stieß die europäischen Alliierten, mit der offensichtlichen Ausnahme Großbritanniens, zunehmend vor den Kopf. Während ein erstarkender Antiamerikanismus auf dem Kontinent zu politischen Zerwürfnissen und Anti-Irakkriegs-Bewegungen führte, schürten politische Kräfte in den USA antieuropäische Ressentiments, wodurch sich die Gräben zwischen den transatlantischen Bündnispartnern immer mehr vertieften (Kagan 2003 u.a.).

Mit der Ablösung von George W. Bush durch den demokratischen US-Präsidenten Barack Obama im Januar 2009, der nicht nur eine Abkehr von einer interventionistisch-missionarischen Außenpolitik einläutete, sondern darüber hinaus eine neue, den Europäern genehmere Rhetorik verwendete, verbesserte sich das Verhältnis zwischen den USA und Europa wieder (vgl. Fröhlich 2012, Vasconcelos/Zaborowski 2009). Doch neue Konfliktpunkte, wie 2013 die Enthüllungen über das amerikanische NSA-Programm Prism und die systematische Sammlung von Informationen über europäische Regierungen und ihre Vertreter, schwächen das historische Bündnis von Neuem. Die USA haben - nicht nur in Europa - Vertrauen verloren und der alleinige Führungsanspruch der Vereinigten Staaten wird immer weniger akzeptiert.

In welcher Weise der Aufstieg Chinas als globale Wirtschaftsmacht die geopolitische Machtkonstellation der transatlan-

\footnotetext{
Mag. Dr. Katharina Götsch ist Politologin und derzeit Projektkoordinatorin an der Universität Wien. Sie arbeitet seit mehreren Jahren als wissenschaftliche Mitarbeiterin und Koordinatorin an internationalen Forschungsprojekten. Ihre Schwerpunkte sind IB-Theorien, Migration, Geopolitik sowie US-Außenpolitik.
} 
tischen Partnerschaft beeinflusst und welche Herausforderungen diese Situation für den Westen - als Bündnis und als vorherrschendes Modell - darstellt, wird im Folgenden behandelt. Darüber hinaus werden einige der wichtigsten Reibungspunkte bzw. Konfliktgegenstände im Verhältnis China - USA - Europa exemplarisch beleuchtet.

\section{Geopolitische Bedeutung der transatlantischen Allianz}

Die Vereinigten Staaten gingen aus dem Zweiten Weltkrieg als ökonomisch-militärische Großmacht hervor und lösten Großbritannien als Hegemon ab (vgl. Friedman 2010). Als die Sowjetunion als zweite Siegermacht über das nationalsozialistische Deutschland ihren Einflussbereich zunehmend erweiterte und sich als systemische Gegenmacht zu den USA in Stellung brachte, schlossen sich die westeuropäischen Staaten zu einem sicherheitspolitischen, ökonomischen und ideologischen Bündnis mit den USA zusammen. Ein gemeinsames System internationaler Organisationen institutionalisierte die Ordnung unter US-amerikanischer Vorherrschaft.

Nach dem Ende des Kalten Krieges verlor das transatlantische Bündnis aufgrund der veränderten Sicherheitslage zu einem gewissen Grad an Bedeutung (vgl. Fröhlich 2012, 7). Europa trieb seine Integration in einer politischen, ökonomischen und kulturellen Union voran und verfolgte das Ziel einer gemeinsamen Identität als Grundlage für eine Europäische Union als einflussreicher Global Player (ebd., Weidenfeld 2013, Hill/ Smith 2011, u.a.).

Schwer geschädigt wurde das transatlantische Bündnis während der Präsidentschaft von George W. Bush, insbesondere als der „Krieg gegen den Terror" in unilateraler Weise ohne UN-Resolution auf den Irak ausgeweitet wurde (Fröhlich 2012, Vasconcelos/Zaborowski 2009, u.a.). Charles Kupchan zufolge ist die amerikanisch-europäische Allianz damit dauerhaft geschädigt worden: „Jetzt, wo die amerikanische und die europäische Sicherheit nicht mehr unteilbar sind, ist der Geist des atlantischen Bündnisses zerstört, selbst wenn es dem Namen nach überleben sollte“ (Kupchan 2003, 9).

\section{Von der bipolaren zur unipolaren Ordnung}

Mit der Implosion des sowjetischen Machtblocks und dem Ende des Kalten Krieges löste sich die jahrzehntelange bipolare internationale Ordnung auf. Es sah danach aus, als würde sich die westliche Weltanschauung mit ihren kapitalistisch-demokratischen Systemen nun auch im ehemaligen Einflussbereich der Sowjetunion durchsetzen und eine globale Demokratisierungswelle in Gang setzen (Brzezinski 2012, Fukuyama 1992 u.a.).

Die Volksrepublik China allerdings konnte den Regimezusammenbruch 1989 durch die gewaltsame und international geächtete Niederschlagung des Volksaufstandes am Tian'anmen-Platz sowie weitgehende wirtschaftliche Reformen verhindern. Seitdem stellt sich dem Westen die Frage, ob bzw. wie das autoritäre und wirtschaftlich äußerst erfolgreiche Regime international integriert werden kann.

Im Bereich der internationalen Beziehungen kristallisierten sich nach den globalen Umbrüchen von 1989 drei große Theorieblöcke heraus, die sich vereinfacht wie folgt darstellen lassen (vgl. Gaiser/Kovač 2012):

- Eine dominante Strömung vertritt die Position, dass sich im Wettstreit der Ideologien Kapitalismus und liberale Demokratie als einzig verbleibende Option durchgesetzt haben. Mit dem Scheitern der Sowjetunion hat sich die historische Alternative einer sozialistischen Ordnung erledigt, wodurch das „Ende der Geschichte“ erreicht wurde (vgl. Fukuyama 1992). Das liberale Modell setzt sich weltweit durch und stellt den Endpunkt der Entwicklung in Form einer New World Order dar, in welcher die früheren Blöcke aufgelöst sind und der Faktor Macht an Bedeutung verliert (vgl. Gaiser/Kovač 2012).

- Eine andere Position sieht eine Ablösung der jahrzehntelangen bipolaren Ordnung durch eine multipolare, da zusätzlich zur US-Macht andere Global Player - u.a. EU, Japan, China - an Bedeutung gewinnen und eine Alleinherrschaft keine Akzeptanz findet (z.B. Kennedy 1992, Mearsheimer 2003, Zakaria 2008). Macht- und Interessenskonflikte werden aus dieser Perspektive weiterhin die internationalen Beziehungen bestimmen.

- Eine besonders starke Strömung in der internationalen Politik konstatiert nach 1989 die alleinige Führungsposition der USA. Mit dem Ende des Kalten Krieges und dem Wegfall des kommunistisch-stalinistischen Blocks wurde aus der bipolaren eine unipolare Weltordnung, in welcher die Vereinigten Staaten mit ihrem europäischen Partner zum einzigen Zentrum werden (Krauthammer 1990 u.a.). Im Gegensatz zu den auch als „Idealisten“ oder „Optimisten“ (Gaiser/Kovač 2012, 51) bezeichneten Theoretikern einer New World Order bleibt die Konzentration von Macht hier das entscheidende Moment.

Genau betrachtet haben sich in den Jahrzehnten seit dem Fall des Eisernen Vorhangs verschiedene Ordnungsphasen abgelöst, die zum einen durch wirtschaftliche Krisen in den Industrieländern eingeleitet wurden und zum anderen durch den Aufstieg früherer Entwicklungsländer als sogenannte emerging powers (Gaiser/Kovač 2012). In der unmittelbaren Folge des sowjetischen Zusammenbruchs und nach den Terroranschlägen auf die USA gab es tatsächlich unipolare Momente, in welchen die Vorherrschaft des Westens bzw. der Vereinigten Staaten gegeben und überwiegend akzeptiert war. Doch in den Zwischenphasen galt eine globale Hegemonie einer unipolaren Macht niemals als unumstritten. Auch das transatlantische Bündnis zwischen USA und Europa durchlebte in dieser Periode Höhen und Tiefen, die zu einem derzeit deutlich abgekühlten und zunehmend distanzierten Verhältnis führten.

\section{Vom Unipolarismus zum Multipolarismus}

In den 1980er und frühen 1990er Jahren etablierte sich Japan als starker wirtschaftlicher Konkurrent für den Westen. Rasant steigende Exporte japanischer Produkte in die USA und gleichzeitige Produktionsverlagerungen amerikanischer Firmen nach 
Asien sorgten in der US-amerikanischen Öffentlichkeit zunehmend für Sorgen über Arbeitsplatzverluste und eine Gefährdung der US-Vorherrschaft (Friedman 2010). Nach der bis heute andauernden Schwächung der japanischen Wirtschaft aufgrund von Finanz- und Wirtschaftskrisen in den 1990er Jahren trat die Volksrepublik China kurz darauf in das Zentrum der internationalen Aufmerksamkeit. Sowohl Russland als auch China wurden als geopolitische Herausforderer gesehen, wobei die Stärken der beiden Mächte unterschiedlicher Art waren und sind. Russland wurde als historischer Gegenspieler des Kalten Krieges aufgrund seiner neuerlich erstarkenden geopolitischen Ambitionen sowie seiner militärischen Stärke mit Argwohn betrachtet (Shleifer/Treisman 2004). China betonte sein Ziel der friedlichen Modernisierung und Entwicklung, brachte zunächst keine ideologischen Alternativen zum westlichen Modell ins Spiel und hielt sich international möglichst bedeckt (Bijian 2005).

Während die USA nach dem 11. September 2001 ihre militärischen, finanziellen und politischen Kapazitäten zunehmend im „Krieg gegen den Terror“ erschöpften, wuchs die chinesische Wirtschaft weiterhin im jährlich zweistelligen Bereich und löste Japan als zweitgrößte Volkswirtschaft ab. Seit die USA und Europa durch die 2007/2008 ausgebrochene Finanz- und Wirtschaftskrise zusätzlich geschwächt sind, wächst die Angst vor dem stetigen Aufstieg Chinas noch mehr. Die Sorge, ob dieser Aufstieg friedlich und im Einklang mit den bestehenden Institutionen und Regeln vor sich gehen wird oder ob die Volksrepublik möglicherweise eine neue, nicht-demokratische Ordnung auf internationaler Ebene etablieren könnte, geht damit einher.

Stefan Fröhlich geht von einem unvermeidlichen Multipolarismus in der Zukunft aus, der sich zum einen durch die bestehende Distanz zwischen den USA und Europa und zum anderen durch den Bedeutungsgewinn Russlands und Chinas ergibt, die ihre Beziehungen nach dem Ende des Kalten Krieges wieder verbessert haben (Fröhlich 2012).

„In today's fragmented world, different rules apply to the great-power rivalry in Asia, the law-based approach of the EU, and the near-anarchy and chaos in the Middle East; and the only common theme is that the US role in all these regions is a diminishing one. (...) Although the current world crisis seems to make multilateral cooperation necessary, confidence in the overall superiority of the Western model in general and the US record in democratization throughout the greater Middle East in particular is - to put it mildly - no longer so pronounced" (Fröhlich 2012, 36-37).

Dieser relative Bedeutungsverlust der amerikanischen Supermacht durch den Schwund ihrer globalen Legitimität und den Aufstieg anderer Mächte (Russland und China) könnte allerdings letztendlich das transatlantische Bündnis zusammenhalten (Fröhlich 2012, 37).

\section{Geopolitische Herausforderungen durch Chinas Aufstieg}

Zehn Jahre nach Beginn des Afghanistankriegs justierte Washington seinen außenpolitischen Schwerpunkt neu und rief das „pazifische Jahrhundert Amerikas“ aus. Im Oktober 2011 verkündete die damalige US-Außenministerin Hillary R. Clinton: „The future of politics will be decided in Asia, not Afghanistan or Iraq, and the United States will be right at the center of the action" (Clinton 2011). Clinton führt aus, welche Rolle die aufstrebenden Staaten Asiens - China, Indien und Indonesien - auf der globalen Ebene spielen, nicht nur aufgrund ihrer geografisch-demografischen Lage, sondern insbesondere aufgrund ihrer wirtschaftlichen Bedeutung als „key engines of the global economy“ (ebd.).

„At a time when the region is building a more mature security and economic architecture to promote stability and prosperity, U.S. commitment there is essential. It will help build that architecture and pay dividends for continued American leadership well into this century, just as our post-World War II commitment to building a comprehensive and lasting transatlantic network of institutions and relationships has paid off many times over - and continues to do so. The time has come for the United States to make similar investments as a Pacific Power (...)" (ebd.).

Sowohl in Europa als auch in den Vereinigten Staaten stellen sich seit einigen Jahren fundamentale Fragen bezüglich der chinesischen Macht: Wird sich China zu einem ,responsible stakeholder' entwickeln und in die internationalen Institutionen, die der Westen nach dem Zweiten Weltkrieg ohne Beteiligung Asiens errichtet hat, eingliedern (Ikenberry 2011, Zakaria 2008)? Oder stellt die Volksrepublik möglichweise eine systemische Alternative dar, deren Markt-Staat-Verhältnis etwa bessere wirtschaftliche Regulierungen und Krisenstrategien ermöglicht? Ergibt sich durch Chinas Aufstieg eine neue bipolare Ordnung oder wird die US-Vorherrschaft gar abgelöst?

Bei der Beantwortung dieser Fragen spielt eine Rolle, wie die parallel zu Chinas Aufstieg vor sich gehende Schwächung der USA und Europas bewertet wird. Geht man davon aus, dass die derzeitige Machtverschiebung zwischen dem Westen und Asien von Dauer sein wird und China die USA wie einst diese Großbritannien als Hegemon ersetzen könnte, so kann bezweifelt werden, ob sich China mit einer Eingliederung in die bestehende westlich-geprägte liberale Ordnung begnügen wird: „Haben im Niedergang begriffene Hegemone irgendeine Berechtigung, eine von ihnen geschaffene Ordnung festzuschreiben, und von ihren Nachfolgern zu erwarten, diese einfach zu übernehmen?" (Clark 2011). Auch Henry Kissinger zweifelt daran, dass sich China in die bestehende internationale Struktur eingliedern wird und der „Westen mit einem immer stärkeren China auskommen und zusammenarbeiten" kann (Kissinger et al. 2012, 33). Verschiedene theoretische und politische Fraktionen innerhalb des transatlantischen Bündnisses propagieren deshalb divergierende Strategien zwischen Containment, Engagement und Congagement (Rudolf in Wacker 2006) zum Umgang mit der zunehmenden globalen Bedeutung Chinas.

\section{Wer beherrscht das 21. Jahrhundert?}

Der Historiker Niall Ferguson diagnostiziert eine ökonomische, politische und moralische Krise der Vereinigten Staaten, die zu einer Ablösung der Vorherrschaft des Westens und gleichzeitig zur Beherrschung des 21. Jahrhunderts durch China führen werde, „weil sich ein übergewichtiges, hochverschuldetes und 
hypersexuelles Amerika und ein dysfunktionales Europa im Niedergang befinden" (Ferguson in Kissinger et al. 2012, 19).

Als Argument für seine Überzeugung von der Vorherrschaft Chinas im 21. Jahrhundert führt er die Tatsache an, dass China während des überwiegenden Teils seiner Geschichte die größte globale Macht darstellte und nun, da sich der Westen im Niedergang befindet, zu diesem Normalzustand zurückkehren werde (ebd.). Auch David Li prognostiziert eine Ablösung des westlich-liberalen Modells durch das von China repräsentierte alternative Gesellschafts- und Wirtschaftsmodell (Li in Kissinger et al. 2012). Die Finanz- und Wirtschaftskrise, die 2007 in den USA ihren Ausgang nahm und sich global fortsetzte, markiere einen historischen Wendepunkt, der das letzte Kapitel der USamerikanischen Vorherrschaft einläutet (ebd.). China habe von den Erfahrungen des Westens gelernt und verbinde nun die ökonomischen Entwicklungslehren mit dem eigenen Wertesystem, in welchem das gesellschaftliche Wohl die persönliche Freiheit des Einzelnen dominiert (ebd.).

Andere Experten verweisen auf die enormen internen Herausforderungen für China (Lampton 2014), innere Widersprüche von sozialen Spannungen bis zu einer nicht nachhaltigen Wirtschaftspolitik, die das Land in der Zukunft an einer weiteren Expansion hindern werde (Zakaria in Kissinger et al. 2012, Zakaria 2008). Diese Ansicht vertreten auch Henry Kissinger und George Friedman (Kissinger et al. 2012, Kissinger 2011, Friedman 2010). Friedman verweist darüber hinaus auf die nachteilige geografische Lage Chinas und die fehlende Tradition als Seemacht, woraus sich starke geopolitische Nachteile ergeben. Die Unmöglichkeit der vorherrschenden Zentrifugalkräfte, die riesige, in unterschiedliche Ethnien geteilten Bevölkerung zusammenzuhalten sowie die internen Asymmetrien und Gegensätze auszugleichen, würde China letztendlich immer wieder daran hindern, zur globalen Macht aufzusteigen (Friedman 2010).

"China is inherently unstable. Whenever it opens its borders to the outside world, the coastal region becomes prosperous, but the vast majority of Chinese in the interior remain impoverished. This leads to tension, conflict, and instability. It also leads to economic decisions made for political reasons, resulting in inefficiency and corruption. This is not the first time that China has opened itself to foreign trade, and it will not be the last time that it becomes unstable as a result. (...) I believe the Chinese cycle will move to its next and inevitable phase in the coming decade" (Friedman 2010, 6-7).

Auch die Bedeutung und die politischen Folgen der Finanzkrise werden unterschiedlich eingeschätzt. So behauptet etwa George Friedman (2010), dass die USA aus der vergangenen Krise gestärkt hervorgegangen sind, während andere Mächte - wie die Europäische Union - sich in Partikularlösungen verlaufen hätten.

"The crisis clarified a number of points. First, the United States is the center of the system. If it has a crisis, everyone has a crisis. Second, the crisis will be worse outside the United States than in the United States. Third, the rest of the world needs the United States to recover if it is to recover" (Friedman 2010, xix).

Diese Position ist auch innerhalb des Westens stark umstritten, so würdigen zahlreiche Experten die rasche und effektive Reak- tion der chinesischen Regierung zu Beginn der Krise. Staatliche Maßnahmen (günstige Kredite für Unternehmen, Konjunkturprogramme etc.) sorgten für ein Abfedern der schlimmsten Krisenerscheinungen und ermöglichten eine stabilisierende Wirkung der chinesischen Wirtschaft für den Westen (vgl. Lee 2011).

\section{Konfliktlinien im geopolitischen Dreieck USA - China - Europa}

In den Beziehungen zwischen China und den USA bzw. Europa sorgen zahlreiche Konfliktfelder für wiederkehrende Auseinandersetzungen. Im Folgenden werden einige dieser Bereiche exemplarisch angeführt, wobei insbesondere die verschiedenen Gewichtungen dieser Fragen innerhalb des Westens hervorgehoben werden.

Zahlreiche Reibungspunkte belasten die asiatisch-westlichen Beziehungen von Handels- und Währungsfragen, über Menschenrechtsverletzungen, den Schutz geistigen Eigentums, Energie und Umwelt bis zu internationalen Rüstungskontrollen und zur Ächtung autoritärer Regime.

\section{Handel und Währung}

Die Europäische Union und die USA sind die wichtigsten Handelspartner Chinas und beide befinden sich mit Peking im Streit um zahlreiche Handelsfragen. Zwischen der EU und China eskalierte etwa 2013 ein Konflikt, in welchem die EU China vorwirft, die europäische Solarbranche durch chinesische Exporte zu Dumpingpreisen zu zerstören und regionale Hersteller vom Markt zu verdrängen. Im Dezember 2013 führte die Europäische Kommission für mehrere Jahre Strafzölle für Solarmodule chinesischer Unternehmen, die sich nicht an die bestehenden Exportregelungen halten, ein. Peking erwiderte diesen Schritt prompt mit erweiterten Prüfungen europäischer Exporte (u.a. Wein, Fahrzeuge etc.).

Ähnliche Konfliktpunkte gibt es auch in den Handelsbeziehungen der USA mit China. Mehrfach schalteten die US-Amerikaner in den vergangenen Jahren die Welthandelsorganisation ein, um Benachteiligungen US-amerikanischer Firmen auf dem chinesischen Markt anzuklagen.

Beide Seiten werfen sich gegenseitig Wirtschaftsprotektionismus und wettbewerbsschädigendes Verhalten vor. Die transatlantischen Partner kritisieren außerdem laufend die Unterbewertung des Yuan. China ist Amerikas größter Gläubiger und hält US-Staatsanleihen im Wert von 1,3 Billionen Dollar. Auch die EU hat gegenüber Peking ein riesiges Handelsbilanzdefizit von 145,8 Milliarden Euro (2012) (vgl. etwa Der Spiegel 2010), wodurch beide Seiten eng miteinander verbunden und voneinander abhängig sind.

\section{Internationale Konflikte}

Hinsichtlich internationaler Konflikte spielt China insbesondere aufgrund seiner Vetomacht im UN-Sicherheitsrat eine zentrale Rolle. Unter Berufung auf das völkerrechtliche Prinzip der Nichteinmischung in die Souveränität anderer Staaten, verhindert 
China immer wieder - gemeinsam mit Russland - die internationale Verurteilung und Sanktionierung autoritärer Regime wie des Iran oder Syriens (vgl. etwa Richter 2013, Zeit Online 2014 u.a.). Häufig steht China hier gemeinsam mit Russland in Opposition zu den westlichen Mächten. In der Auseinandersetzung der internationalen Gemeinschaft mit dem nordkoreanischen Regime um dessen Atomprogramm versucht China, als einziger Verbündeter der Kim-Diktatur, eine Vermittlerposition einzunehmen. Das wichtigste Ziel der Volksrepublik ist die Stabilität der Region, weshalb China eine Eskalation des Konflikts zwischen Nordkorea auf der einen Seite sowie den USA und seinem südkoreanischen Partner auf der anderen Seite unbedingt zu verhindern versucht (Bajoria/Xu 2014). Durch wirtschaftliche und humanitäre Hilfe sowie bilateralen und multilateralen Druck auf das nordkoreanische Regime bemüht sich China um eine Lösung des Nuklearproblems in Korea.

\section{Energie und Kooperation}

Die rasante Modernisierung und industrielle Expansion der chinesischen Wirtschaft führt zu einer enormen Energienachfrage, deren Deckung die Volksrepublik durch bilaterale Verträge und Investitionen abzusichern versucht. Chinas Investitionen in ressourcenreiche Länder in Afrika, Lateinamerika und im Nahen Osten (oftmals in Form von Entwicklungshilfen, vgl. Hilton 2013) ist dem Westen, der hier historische Ansprüche stellt, ein Dorn im Auge. Die strategischen Beziehungen Chinas, u.a. im Rüstungsbereich, zu international isolierten und geächteten Regimen wie dem Iran, Venezuela oder Nordkorea verursachen bei den transatlantischen Partnern Sorgen, die Volksrepublik verbünde sich mit strategischen Gegnern des Westens zu einer Allianz (vgl. Hilton 2013).

\section{Herausforderungen für die transatlantische Gemeinschaft}

Sowohl die westlichen Mächte als auch China sind sich im Klaren darüber, dass gewisse Herausforderungen und Konflikte nur unter Einbezug aller großen Akteure gemeistert werden können. Darüber hinaus sind die wirtschaftlichen Verflechtungen und Dependenzen zwischen den Regionen dergestalt, dass eine Kooperation zwischen den potenziellen Rivalen eine existenzielle Frage darstellt. Für China ist es ebenfalls von größter Notwendigkeit, mit den westlichen Staaten und den internationalen Institutionen friedlich zusammenzuarbeiten. Nur auf diese Weise kann das Wirtschaftswachstum und in Folge die innere Stabilisierung und Weiterentwicklung des Landes garantiert werden.

Eberhard Sandschneider vom Forschungsinstitut der Deutschen Gesellschaft für Auswärtige Politik beschreibt Chinas Handlungsoptionen als Manövrieren zwischen externen und internen Faktoren, als Balanceakt: „Chinas Führung muss ihren Stabilitätskurs in einem nach wie vor hochgradig von Unsicherheiten geprägten Umfeld steuern und gleichzeitig seinen wachsenden internationalen Einfluss erfolgreich managen" (Sandschneider 2012, 44).
Die westlichen Staaten der transatlantischen Gemeinschaft teilen eine Reihe von Konfliktpunkten und Befürchtungen in den Beziehungen zu China: Der internationale Umgang mit der Klimaerwärmung, das Stützen der krisengeschüttelten Weltwirtschaft, die Befriedung internationaler Konflikte - die USA und Europa haben ein gemeinsames Interesse, diese Herausforderungen gemeinsam mit den asiatischen Mächten, allen voran China, zu lösen. Latente Konflikte innerhalb des westlichen Bündnisses offenbaren sich allerdings auch in der Auseinandersetzung mit dem globalen Aufstieg Chinas. Die USA erheben in der pazifischen Region einen sicherheitspolitischen Führungsanspruch. Auf globaler Ebene sind die USA darüber hinaus nicht bereit, ihre im Laufe des 20. Jahrhunderts errungene Vormachtstellung aufzugeben. Die Ambitionen Chinas, seinen Einfluss zu festigen und auszuweiten - mittels ökonomischer Methoden oder militärischer Stärke -, betrachtet Washington als Angriff auf die US-Hegemonie. Da die USA gewichtige geopolitische Interessen nicht nur in Asien - haben, dominiert in der theoretischen und politischen Auseinandersetzung mit China die Abwehrhaltung gegenüber der militärischen Expansion und die Befürchtung, von China überholt und abgelöst zu werden.

Europa dagegen verfolgt keine geopolitischen Strategien im Pazifik und ist deshalb in erster Linie an diplomatisch-wirtschaftlichen Beziehungen mit China interessiert (Fröhlich 2012). Die seit Jahren steigenden Militärausgaben der chinesischen Regierung, die in den USA die Debatten über Asien dominieren, erwecken in den EU-Ländern kaum Befürchtungen vor einem aggressiven China. Diese Bedenken hegt Europa vielmehr gegenüber den Ambitionen Russlands in dessen Nachbarschaftsregionen.

Die unterschiedliche Gewichtung der Debatte über Chinas globale Rolle birgt ein gewisses Risiko für die Transatlantikpartnerschaft. Sollten sich die westlich-chinesischen Beziehungen in der Zukunft verschlechtern und eine militärische Dimension erreichen, stünde Europa (das in dieser Frage selbst nicht geeint ist), das „bei der Debatte um die künftige Rolle Chinas immer noch in schöner Regelmäßigkeit zwischen nackter Angst und schierer Begeisterung" schwankt, zwischen den Stühlen (Sandschneider 2012, 45).

„Although it is hard to envisage Europeans actively taking China's side in such an eventuality, it is certainly conceivable that the EU could split over how to respond - or adopt a position of nervous neutralism. In either scenario, the US would view Europe's vacillations as a profound betrayal" (Gowan/Kundnani 2014).

Die Frage, wie der Westen mit dem Aufstieg Chinas umgehen soll, wird nach Sandschneider vor allem deshalb so brisant und hitzig debattiert, da „es sich eben um ein kommunistisches Land handelt, das mit anderen Wertvorstellungen und politischen Ordnungsprinzipien seine globale Machtstellung ausbaut" (ebd.). Aus diesem Grund empfiehlt sich von Seiten der transatlantischen Staaten ein flexibler Pragmatismus im Umgang mit dem legitimen Wachstum Chinas (ebd.), bei dem sich zum einen der Westen für die Bedürfnisse und Anforderungen eines erstarkten ehemaligen Entwicklungslandes öffnet und sich den veränderten Machtverhältnissen anpasst und sich zum anderen die aufsteigenden Länder - allen voran China - innerhalb der internationalen Ordnung als verantwortungsvolle Akteure weiterentwickeln und integrieren können. 
Für die transatlantische Allianz, die ohnehin geschwächt und deutlich abgekühlt ist unter anderem durch ideologisch-politische Konflikte (nicht nur während der Bush-Administration), diplomatische Zerwürfnisse über die NSA-Aktivitäten und die Rolle der US-Regierung hierbei sowie andere Diskrepanzen, ist der Umgang mit Chinas Aufstieg ein Prüfstein. Nur wenn der Westen die geopolitischen Verschiebungen und die Erosion des unipolaren Moments zu akzeptieren bereit ist, kann China als gleichberechtigter Partner in die internationale Gemeinschaft integriert werden - zum Nutzen beider Seiten.

\section{Referenzen}

Bajoria, Jayshree/Xu, Beina (2014). The China-North Korea Relationship, Backgrounder, Council on Foreign Relations: http://www.cfr.org/china/china-north-korea-relationship/ p11097 (zuletzt abgerufen am 30.03.2014).

Bijian, Zheng (2005). China's "Peaceful Rise" to Great-Power Status, in: Foreign Affairs, Sept./Okt. 2005: http://www. foreignaffairs.com/articles/61015/zheng-bijian/chinas-peacefulrise-to-great-power-status (zuletzt abgerufen am 01.12.2013).

Brzezinski, Zbigniew (2010). Strategic vision. America and the crisis of global power, Basic Books, New York.

Clark, Ian (2011). Hegemony in International Society, Oxford University Press, New York.

Clinton, Hillary R. (2011). America's Pacific Century, in: Foreign Policy Magazine, October 11, 2011: http://www.foreignpolicy. com/articles/2011/10/11/americas_pacific_century (zuletzt abgerufen am 23.12.2013).

Der Spiegel (2010). Handelsbilanz-Defizit: China importiert zu viel, Spiegel Online, 10.04.2010: http://www.spiegel.de/ wirtschaft/soziales/handelsbilanz-defizit-china-importiert-zuviel-a-688255.html (zuletzt abgerufen am 10.02.2014).

Deudney, Daniel et al. (2011). Global Shift. How the West should respond to the rise of China. Transatlantic Academy.

Friedman, George (2010). The Next 100 Years. A Forecast for the $21^{\text {st }}$ Century, Anchor Books, New York.

Fröhlich, Stefan (2012). The New Geopolitics of Transatlantic Relations. Coordinated Responses to Common Dangers, The John Hopkins University Press, Baltimore.

Fukuyama, Francis (1992). The end of history and the last man, Free Press, New York.

Gaiser, Laris/Kovač, Igor (2012). From Bipolarity to Bipolarity: International Relations Repeating Again, in: Journal of Global Policy and Governance, Vol. 1, Issue 1 2012, 49-63.

Gowan, Richard/Kundnani, Hans (2014). Why Europe can't leave Asia to the US: http://ecfr.eu/content/entry/commentary_ why_europe_cant_leave_asia_to_the_us237 (zuletzt abgerufen am 14.01.2014).

Hill, Christopher/Smith, Michael (Hg.) (2013). International Relations and the European Union, Oxford University Press, New York.
Hilton, Isabel (2013). China in Latin America: Hegemonic Challenge?, Norwegian Peacebuilding Resource Center, February 2013: http://www.peacebuilding.no/var/ezflow_site/ storage/original/application/26ff1a0cc3c0b6d5692c8afbc054a ad9.pdf (zuletzt abgerufen am 20.01.2014).

Ikenberry, G. John (2011). Liberal Leviathan.The Origins, Crisis, and Transformation of the American World Order, Princeton University Press, Princeton/Oxford.

Kagan, Robert (2003). Macht und Ohnmacht. Amerika und Europa in der neuen Weltordnung, Siedler Verlag, Berlin.

Kennedy, Paul (1992). In Vorbereitung auf das 21. Jahrhundert, Bertelsmann Verlag, München.

Kissinger, Henry (2011). China. Zwischen Tradition und Herausforderung, C. Bertelsmann Verlag, München.

Kissinger, Henry/Zakaria, Fareed/Ferguson, Niall/Li, David Daokui (2012).Wird China das 21. Jahrhundert beherrschen? Eine Debatte, Pantheon Verlag, München.

Krauthammer, Charles (1990). The Unipolar Moment, in: Foreign Affairs, Vol. 70, 1990/91, 23-33.

Kupchan, Charles (2003). Die europäische Herausforderung. Vom Ende der Vorherrschaft Amerikas, Rowohlt, Berlin.

Lampton, David M. (2014). How China is ruled. Why It's Getting Harder for Beijing to Govern, in: Foreign Affairs, Jan./Feb. Issue 2014.

Lee, Felix (2011). Die Gewinner der Krise. Was der Westen von China lernen kann, Rotbuch-Verlag, Berlin.

Mearsheimer, John J. (2003). The Tragedy of Great Power Politics, W. W. Norton \& Company, New York.

Richter, Steffen (2013). Syrien-Krieg. Warum China gegen Assad nicht mitmacht, Die Zeit, 12.09.2013: http://www.zeit.de/politik/ ausland/2013-09/syrien-china (zuletzt abgerufen am 15.03.2014).

Ross, Robert S. (2005). Assessing the China Threat, The National Interest: http://nationalinterest.org/article/assessing-the-chinathreat-345 (zuletzt abgerufen am 12.03.2014).

Sandschneider, Eberhard (2012). Gestaltungsmacht China. Mit Kooperation statt Konfrontation zur Ko-Evolution, in: IP Internationale Politik, März/April 2012.

Seitz, Konrad (2000). China. Eine Weltmacht kehrt zurück, Siedler Verlag, Berlin.

Shambaugh, David (2013). China Goes Global: The Partial Power, Oxford University Press, New York.

Shleifer, Andre/Treisman, Daniel (2004). A Normal Country: Rethinking Russia, in: Foreign Affairs, März/April 2004: http:// www.foreignaffairs.com/articles/59707/andrei-shleifer-and-danieltreisman/a-normal-country (zuletzt abgerufen am 20.12.2014).

Terhalle, Maximilian (2012). Amerika oder Asien? Wie der alte Hegemon mit der neuen Supermacht China kooperieren könnte, in: IP Internationale Politik Online: https://zeitschrift-ip.dgap.org/ de/article/amerika-oder-asien (zuletzt abgerufen am 10.01.2014).

Tisdall, Simon (2014). China's military presence is growing. Does a superpower collision loom?, The Guardian, 01.01.2014: 
http://www.theguardian.com/world/2014/jan/01/chinamilitary-presence-superpower-collision-japan (zuletzt abgerufen am 10.02.2014).

Vasconcelos, Álvaro de/Zaborowski, Marcin (2009)(Hg.). The Obama Moment. European and American Perspectives, EU Institute for Security Studies, Paris.

Wacker, Gudrun (2006)(Hg.). Chinas Aufstieg: Rückkehr der Geopolitik?, SWP-Studie, Februar 2006, Berlin.
Weidenfeld, Werner (2013). Die Europäische Union, UTB, Stuttgart.

Zakaria, Fareed (2008). Der Aufstieg der Anderen. Das postamerikanische Zeitalter, Siedler Verlag, München.

Zeit Online (2014). UN-Sicherheitsrat. Russland und China blockieren erneut Syrien-Resolution, Zeit Online, 22.05.2014: http://www.zeit.de/politik/ausland/2014-05/russland-chinasyrien-resolution (zuletzt abgerufen am 24.05.2014).

\title{
The "Tight Oil Revolution" and its Consequences for the European Union: A "Wake-up Call" for its Neglected Energy Security
}

\section{Susanne Peters*}

\begin{abstract}
This article focuses on the consequences for Europe of the "tight oil revolution" and the projected "risk aversion" strategy of the United States in the Middle East following its recent energy independence. No longer able to free-ride on the back of US military control in the region, the EU is ill prepared for any potential supply crisis and must rely on an inefficient, incoherent and all too market-oriented energy security. The article concludes that the EU should use the newly created geopolitical constellation as a wake-up call to, in the medium term, design a grand strategy for energy security within its foreign policy to better provide favorable investment climates in producer states, and, in the long term, to transform itself into a low-carbon society.
\end{abstract}

Keywords: Oil, European Union, energy security

Stichworte: Erdöl, Europäische Union, Energiesicherheit

A ccess to and availability of affordable oil is a key factor in the smooth functioning of any industrialized society of the world economy, particularly in oil-dependent nations like the United States and its Western allies, as well as Asian nations. Since the end of World War II, the US has exerted its military influence in the Middle East to prevent a hostile power from gaining control of the Persian Gulf, with the inevitable skyrocketing of oil prices that would follow. As the closest allies to the US, the heavily oil-dependent European states could always count on free-riding on the US' geopolitical ${ }^{1}$ engagement in this region. But in the wake of a general withdrawal from its traditional role as a world policeman and the effects of its "tight oil revolution", the US recently made its first moves to relax its strategic engagement in the Middle East and will revert to a policy of "risk aversion" in the event of a crisis in the Middle East. On the basis of this assumption, the article will argue that this US foreign policy shift is a concern for the Europeans, and that the EU has pursued an incomprehensive and incoherent energy security strategy, too market-driven to cope with future challenges. These challenges will be posed by potential supply outages due to a disruption crisis and the projected substantial reductions of deliveries from its traditional main suppliers. The article will further reject the option of making the current

* $\quad$ PhD, Kent State University and Webster University Geneva, Lecturer. This article has been double-blind peer-reviewed.

1 For a discussion of the concept of "geopolitics" see Peters and Westphal, 2013, pp. 93-96.
EU energy security policy more robust by militarizing it in the context of the EU Common Security and Defense Policy. While it does not recommend engaging in any kind of realist energy strategy, this paper will conclude that the EU should use this newly created geopolitical constellation as a wake-up call to design a grand energy strategy in the medium term which makes use of the EU's political and economic power to provide a business-climate-fostering investment and trading contracts with EU oil companies in producer states. In the long term, the EU must shed its oil import dependency by transforming into a low-carbon society. The article will begin by describing the geopolitical effects of the tight oil revolution.

\section{The tight oil revolution ${ }^{2}$ and the consequences for the United States}

The emergence of this unexpected oil glut by the United States is the result of political, economic and technological factors. ${ }^{3}$ Two exploration techniques, known for decades - hydraulic fracturing and horizontal drilling - were combined to make the

2 Tight oil, or "Light tight oil" is conventional oil trapped in fine rock pores or cracks where it cannot escape without stimulation. In contrast "Shale oil" is synthetic oil from kerogene extracted and mined from oil shales, transformed with high energy input.

3 For background on the shale and tight oil revolution see Peters and Zittel, 2014 , forthcoming. 